\title{
Modular OrCAD Simulation Approach in Teaching Power Electronics
}

\author{
Mohamed Darwish \\ Brunel University, U.K. \\ Mohamed.darwish@brunel.ac.uk
}

\author{
Christos Marouchos \\ Cyprus University of Technology, Cyprus \\ Christos.marouchos@cut.ac.cy
}

\begin{abstract}
In this paper the authors present a new technique which can be used in simulating multi power electronic circuits at the same time in a very accurate way without the worries of the circuit analysis being diverted during the simulation. In this technique each circuits is simulated alone and the output current/voltage waveform(s) of this circuit is presented as a 'wave-form' generator. Then the final multi circuit design will be a collection of these 'wave-form' generators. This technique can be used in teaching power electronics system design for undergraduate as well as postgraduate levels and can also be used in the industry. A UPS system is used as an example in demonstrating this technique but this approach could equally be applicable to other multi circuit design configurations.
\end{abstract}

Index Terms-Power Electronics, Simulation in Power Electronics, Education in Power Electronics, OrCAD, PSPICE Simulation, UPS, Modular Simulation.

\section{INTRODUCTION}

Teaching power electronics with the help of simulation packages is very essential nowadays in order to explain/examine the concept of circuit topologies (inverters / ac voltage controllers/dc-dc converters, etc.). The main challenge is when students or engineers try to simulate multi circuits in order to examine the overall performance or efficiency of a system. For example, a typical Uninterruptible Power Supply (UPS) system could contain a rectifier, inverter, battery charger, transformers, filters, etc. Even the most experience engineer with excellent knowledge in simulation packages will find such task very challenging and he will definitely run into the usual simulation 'convergence problem' message. This may not be the case in some packages like MatLab, however MatLab is a system level simulation and not a component level simulation software like OrCad. This challenge (simulating multi circuits together) does not exist when it comes to actually building them in the lab; as the diversion issue is only related to the finite calculation at very small time-steps. In this paper, multi power electronic circuits (connected together) will be simulated using a modular approach which implements wave-form generators. The output of each circuit is presented as a waveform generator and then the overall system (the multi circuits) becomes a collect of waveform generators connected to the final load. The multi circuits could also be presented as a single waveform generator and can be used as a single block in a bigger and more complicated system.

\section{SIMULATION OF INDIVIDUAL PARTS OF POWER ELECTRONIC CIRCUITS}

A typical power electronic system may contain a number of individual circuits/blocks. Each circuit/block could be a converter that converts the power from one shape to another (AC/DC, DC/AC, DC/DC, AC/AC) or it could also be a transformer, passive or active filters. In order to have a clear idea of such system, a double-conversion UPS system is used as an example of this modular system as shown in Fig. 1.

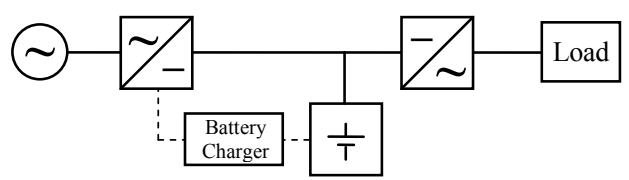

Fig. 1. A typical double conversion UPS System

If such a system to be simulated in OrCAD, then each of the blocks needs to be simulated, which is a straight forward process) and then all circuits need to be connected together, which is not a straight forward process. The reason because as you start connect the output of each circuit to the input of the following circuit, a convergence error similar to the one shown in Fig. 2 will appear straight away.

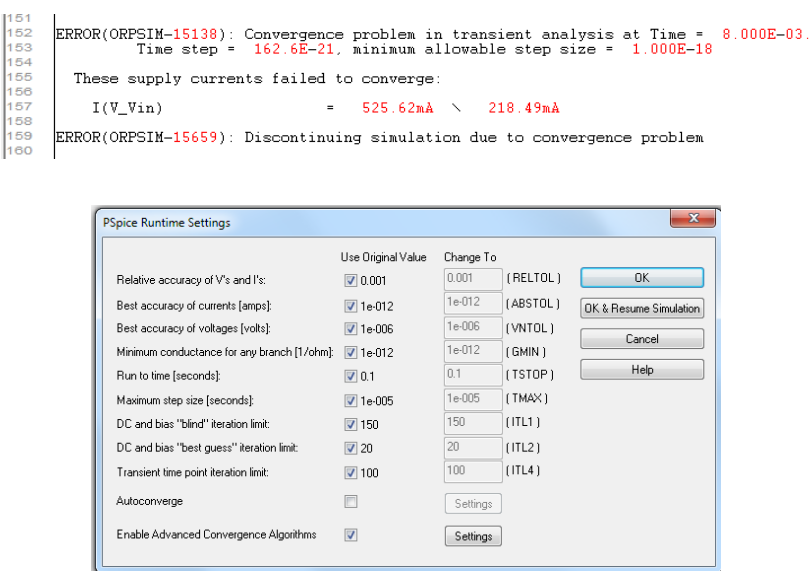

Fig. 2: Convergence error when multi circuits are simulated 
The reason behind the convergence problem message is that the OrCAD-PSpice is trying to solve a set of non-linear equations that describes the circuit behaviour. The solution involves the use of iterative techniques like Newton-Raphson algorithm. In such algorithm, OrCAD-PSpice starts with initial guessing to the solution and then iterate the solution to improve it until the final voltages and currents converge to the same results (within very small variation). In multi circuits simulations, or sometimes even in single circuit simulations, PSpice cannot converge and cannot find a solution to the multi circuit simulation. In this case NewtonRaphson repeating series cannot converge into a consistent set of voltages and currents. This phenomenon has been discussed recently in a number of literatures [1-4]. In this example (UPS) the first two stages are considered, the $\mathrm{AC} / \mathrm{DC}$ rectifier and the DC/AC inverter. Each stage is simulated as individual circuit and then the two are simulated together to identify the problem. The two circuits are then simulated together again with the utilisation of 'waveform generator'.

Fig. 3 shows a simple rectifier circuit simulated in OrCADPSpice using four diodes and a smoothing capacitor. The supply voltage was selected to be $100 \mathrm{~V}$ maximum but any other values could also be selected as long the diode ratings are compatible with the supply voltage and load current.

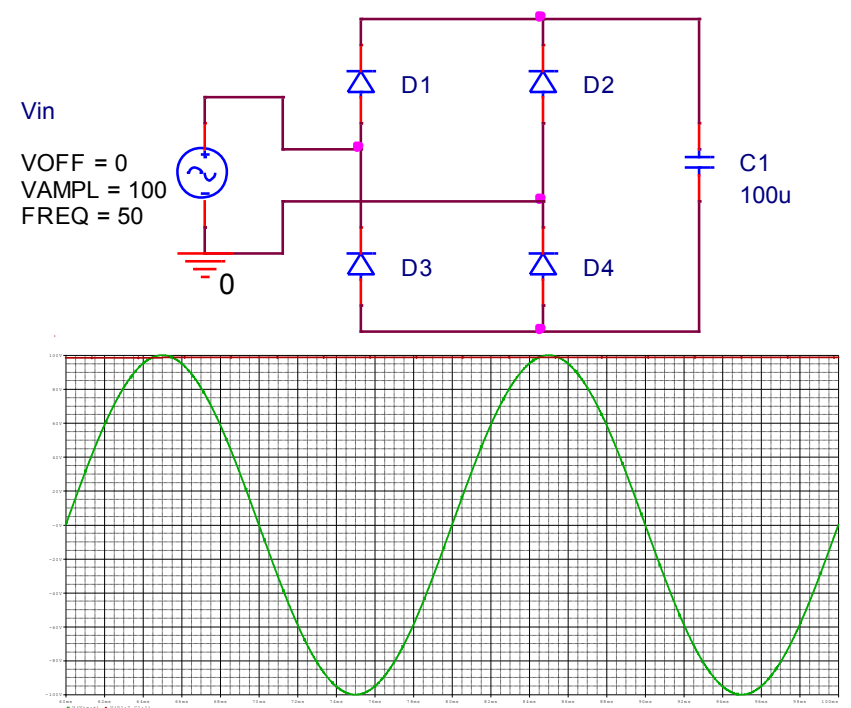

Fig. 3: The rectifier stage in the UPS system (Circuit diagrams and input and output voltages)

The simulation of such circuit is a straight forward and just some attention should be given to the "Maximum step size" option so that the waveforms are nice and smooth. A typical value for the maximum step sixe is $10 \mu \mathrm{s}$. A smaller value could be selected but in this case the simulation takes longer time and also if the maximum step size is too small a convergence problem may occur.

The inverter circuit is then simulated using a DC source (not the output of the rectifier circuit). To simplify the example, a single Pulse Width Modulation (PWM) is used to control the four inverter 'ideal' switches. The timing of the control pulses are generated from VPULSE remotely using 'vcc' (renamed as V+ and V- in this example as shown in Fig. $4)$.
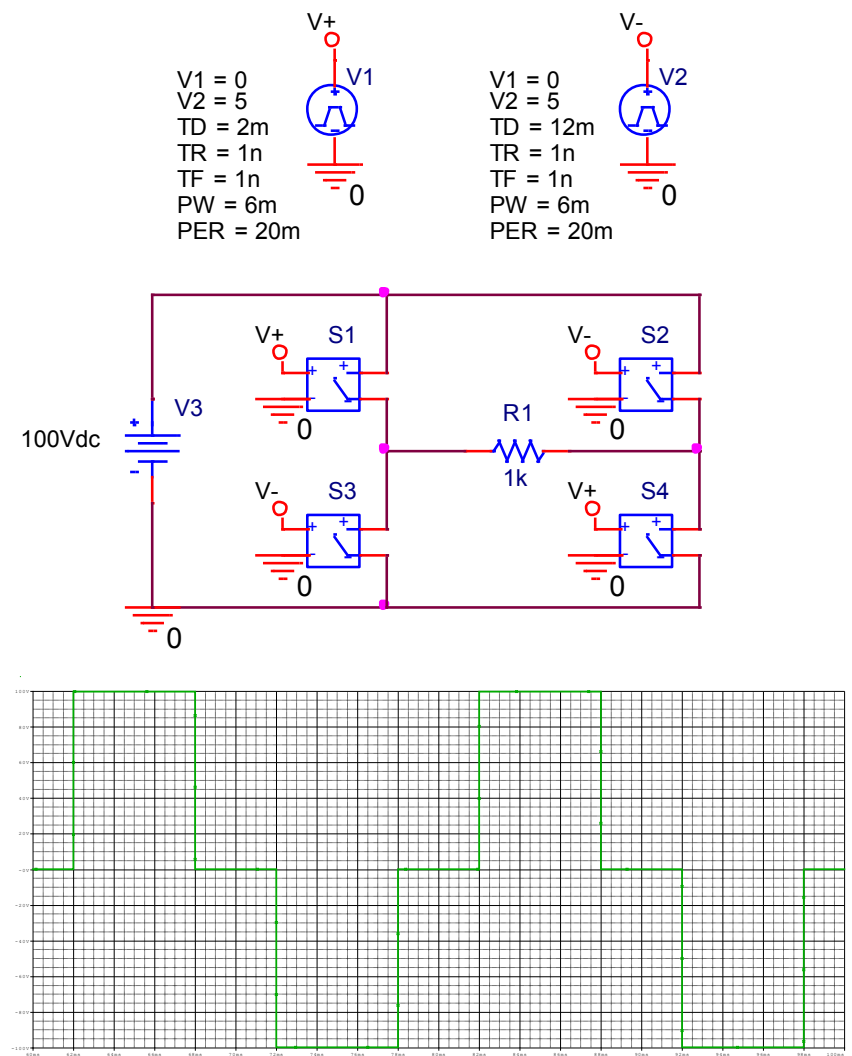

Fig. 4: The inverter stage in the UPS system (Circuit diagrams and output voltage)

A study of the inverter output can then be carried out in terms of harmonic and efficiency (after replacing the ideal switches with semiconductors). Now the challenge begins when the two circuits are to be simulated together in order to see the behaviour of both of them. For example a study of the input current harmonics of the rectifier circuit in conjunction with the output voltage harmonics of the inverter circuit. Or the efficiency of the two converters together (the final output power over the total input power). Or the power factor of the combined circuits. As soon as you integrate the two converters in one diagram and try to simulate the overall circuit, the convergence problem message will appear. The programme used in simulation assumes some initial condition for $x_{0}$. Where $x_{0}$ represents the current, voltage or any 
variable needs to be solved. The next value of $x,\left(x_{1}\right)$ is then evaluated by:

$$
\begin{aligned}
& x_{1}=x_{0}-\frac{f\left(x_{0}\right)}{f^{\prime}\left(x_{0}\right)} \\
& \text { and } x_{n+1}=x_{n}-\frac{f\left(x_{n}\right)}{f^{\prime}\left(x_{n}\right)}
\end{aligned}
$$

The process is carried out until a satisfactory sufficient and accurate value of $x$ is achieved. The conflict could happen between the two circuits during the simulation. One circuit could require positive variation in $\Delta x$, while the other circuit could require negative variation in $\Delta x$. As a result of that the convergence problem occurs. Fig. 5 shown the two circuits together and the error message associated with the analysis.

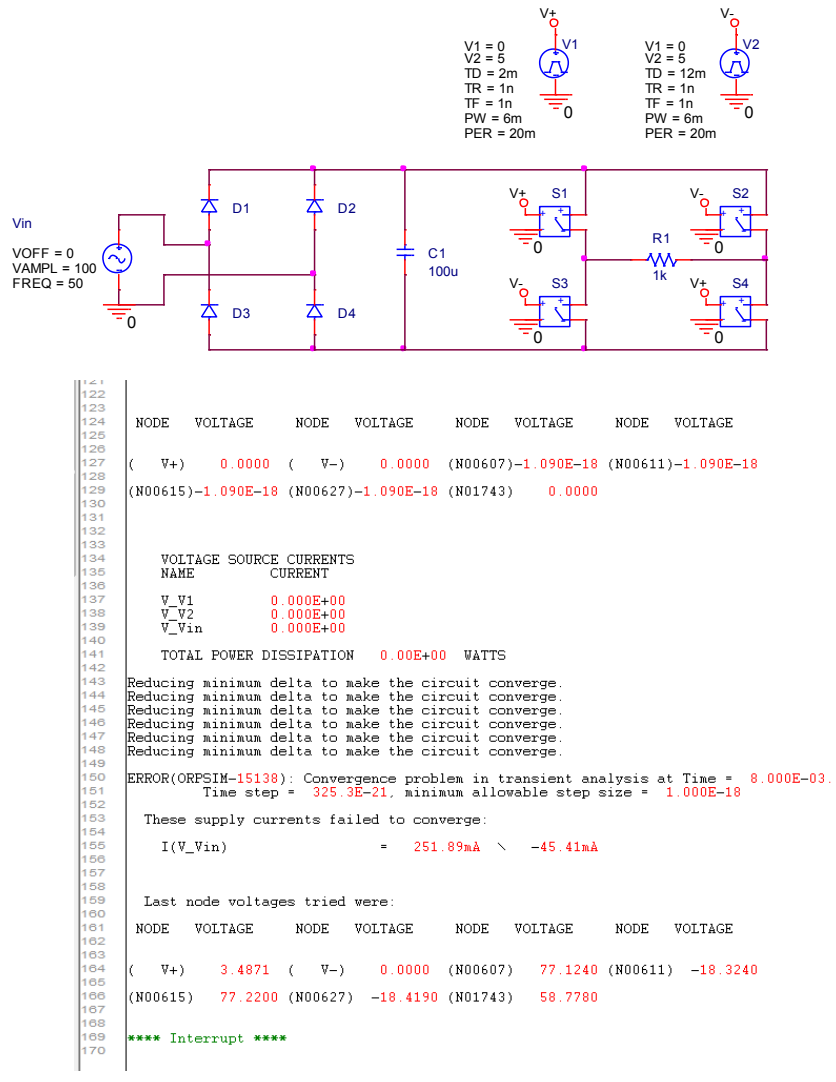

Fig. 5: Combined rectifier/inverter circuit together with the convergence error problem

It can be seen from the convergence problem message that the time step required in the analysis is $325.3 \mathrm{E}-21$ second while the minimum allowable step size is $1.000 \mathrm{E}-18$. The maximum and minimum step sizes can be adjusted manually in an attempt to overcome this problem but apart from the huge time consumed (or wasted) the problem will stay the same. In order to overcome such limitation a waveform generator will be used to break down the two circuits, but yet connect them together as shown in the following section. So far for simplicity only two circuits are considered here (rectifier and inverter), however the same can also be applied to a much larger circuit as will be illustrated later on in the paper.

\section{SIMULATION OF MULTI POWER ELECTRONIC CIRCUITS CONNECTED TOGETHER}

To simulate the two circuits (rectifier and inverter), first the rectifier circuit is simulated alone (like the one shown in Fig. 3 ). The output from the rectifier circuit is saved in file (any format will do). Then a waveform generator is linked to that file. In OrCad the waveform generator is:

VPWL_F_RE_FOREVER and it has the following icon:

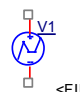

The name $<$ FILE $>$ is linked to the file which contains the voltage output of the rectifier circuit. The rectifier inverter circuits will now look like the one shown in Fig. 6.
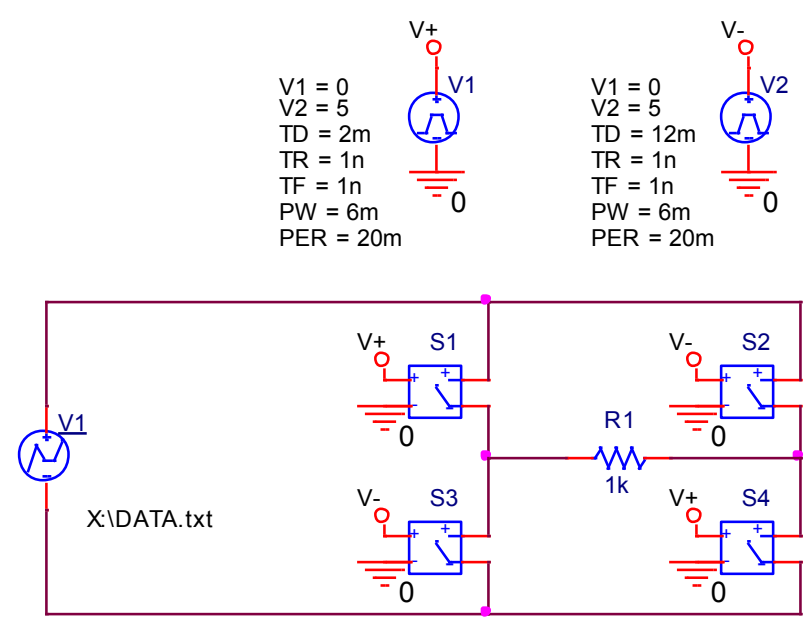

Fig. 6: Inverter circuit connected to a modular rectifier circuit

The waveform generator shown in Fig. 6 is simply the:

'VPWL_F_RE_FOREVER' and is linked to a txt file called 'DATA' which contains simple two coulombs ( $t$ and $v$ ) of the output stage of the rectifier circuit. In this case the simulation only deals with one variable which is the inverter equations and it freezes (or it does not have to deal with) the other variable 'rectifier' at the same time. The final output from the inverter circuit will be the output from the two converters (rectifier and inverter circuits). Further studies can then be carried out at the combined circuit like harmonic analysis, power factor investigation, etc. The property of 'VPWL_F_RE_FOREVER' is shown in Fig. 7 where the name of the text file is inserted under the FILE field

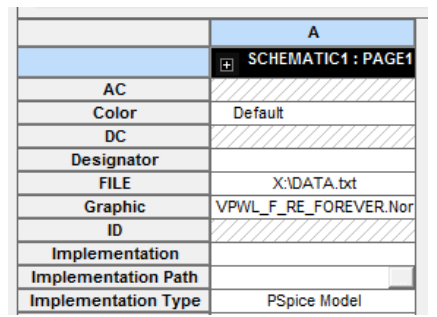

Fig. 7: property of 'VPWL_F_RE_FOREVER 


\section{Simulation OF A UPS SYStem}

In order to demonstrate the effectiveness of the modular technique, a UPS system which contains a rectifier, an inverter, a battery charger and a battery was simulated using this modular approach as shown in Fig.8. The rectifier circuit is simulated and the output waveform is then stored in a file called 'Rectifier Circuit'. A waveform generator is then linked to that data file and used with a battery and battery charger circuit. The output from of this circuit is then saved in another data file and another waveform generator is linked to this data file and simulated together with an inverter circuit. The Output from the Inverter and the second waveform generator is the final output of the UPS. Filter circuits and/or bypass switches could be added in the same manor.

The UPS system could be as simple or as complicated as it could, because the modular approach can easily be used to simulate other parts and then integrate them within the main UPS system as separate waveform generators. The final UPS could be presented as a single waveform generator connected to a load. Obviously in order to carry out any studies or investigation within any part of the UPS system then you have to go back to this part within the modular structure and carry out the study or the investigation. For example, a high frequency UPS system (in order to reduce the size of the magnetices) was simulated. In order to see the waveform at the input of the inverter the markers has to be inserted at the output of the modular system just before the inverter stage as shown in the upper trace of Fig. 9. The final output of the UPS system can also be displayed before any filters as the one shown in the lower trace of Fig. 9.

\section{CONCLUSIONS}

Simulating power electronic circuits is a vital educational tool in teaching power electronics. However, sometimes this tool could be very frustrated and could have the opposite effect. This is usually happening when a complicated circuit is being simulated and the very unpleasant common message "convergence problem" occurs. In this paper a new modular simulation system is introduced. The system is based on simulating the first part of the complicated circuit and then presents the output as a waveform generator, then uses this waveform generator in conjunction with the second part in order to generate the output of the next stage which is then presented as another waveform generator, and so on.

It is worth mentioning that in real life (practical circuits) the conversion problem does not occur. So the proposed modular solution is mainly for a 'theoretical' problem which does not exist in practical situations but still needs to be simulated.

\section{REFERENCES}

[1] "Exploring the Nature of Spice Convergence Problems," http://www.google.co.uk/url? sa $=t \& r c t=j \& q=\& \operatorname{ssrc}=s \&$ frm $=1 \&$ sourc e=web\&cd=3\&ved $=0 C E M O F j A C \& u r l=h t t p \% 3 A \% 2 F \% 2 F w w w . c s e . c$ uhk.edu.hk\%2F phwl\%2Fmt\%2Fpublic\%2Farchives $\% 2$ Fold $\% 2 F$ ceg 3470\%2FPSpice-

$\underline{\text { ConvergenceOnly.pdf\&ei }=y I 5 b U 6 C p B s r J O Z L r g M A B \& u s g=A F Q j C N}$ HaIzUCsVxcihGfnR5KeJitZ1dwgw

Acccessed in April 2014

[2] Wen Ding ; Gaofeng Wang ; Xi Chen: "Accuracy-controlled convergence criterion for full wave simulation", IEEE International Wireless Symposium (IWS), 2013 , Page(s): $1-4$.

[3] Rogulin, L.Y. ; Gubkina, V.R.: "Simulation of power electronic devices using OrCAD software tool", $14^{\text {th }}$ International Conference on Micro/Nanotechnologies and Electron Devices (EDM), 2013, Page(s): $333-335$.

[4] Darwish, M. ; Marouchos, C.: "Simulation levels in teaching power electronics", 48th International Universities Power Engineering Conference (UPEC), 2013, Page(s): 1-5. 


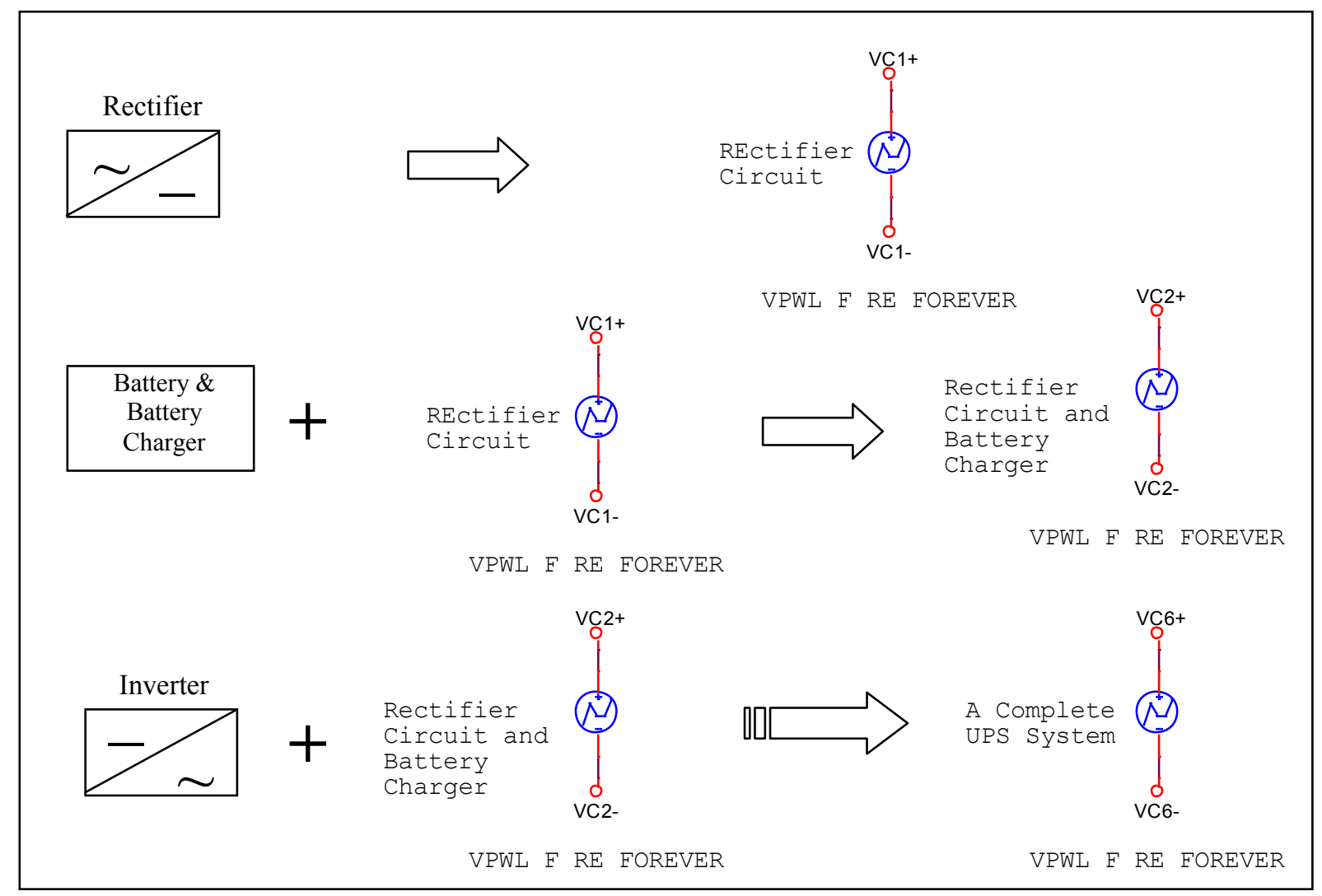

Fig. 8: An Example of Modular Simulation of a Double conversion UPS System

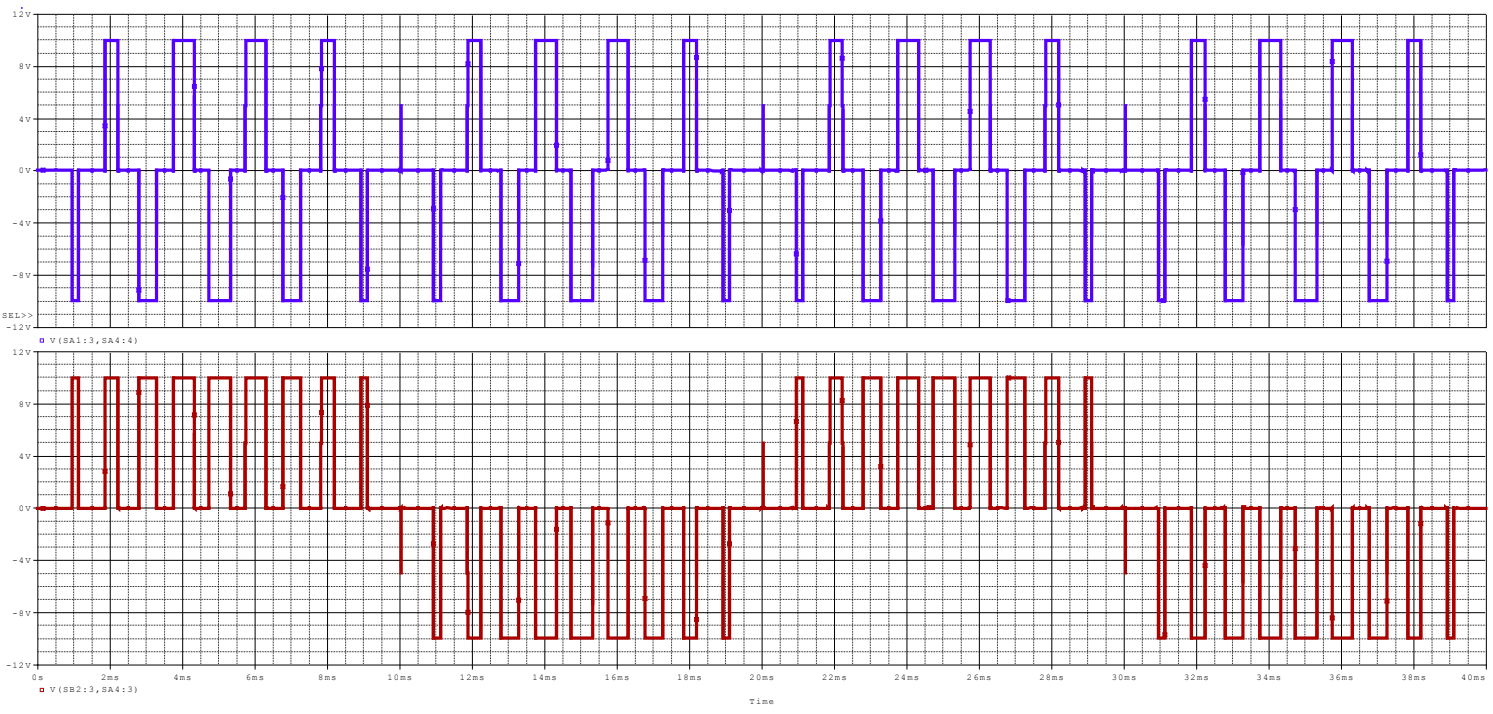

Fig. 9: Simulated waveforms of High Frequency UPS system generated by modular technique 